\title{
Brainstem reticular formation lesions: Amnestic effects on learned habits in the rat*
}

\author{
ROBERT THOMPSON \\ Louisiana State University, Baton Rouge, Louisiana 70803 \\ and \\ B. MICHAEL THORNE \\ Mississippi State University, University, Mississippi 38677
}

\begin{abstract}
Adult albino rats, previously overtrained on a brightness and a pattern discrimination habit, sustained bilateral lesions to the brainstem reticular formation and were subsequently tested for retention. Those groups receiving lesions to the basolateral mesencephalic reticular formation or to the paramedial portion of the reticular formation at either the dimesencephalic juncture, mes-metencephalic juncture, or rostral pontine levels exhibited significant losses in retention of the pattern habit. Lesions destroying either the dorsomedial mesencephalic reticular formation or brainstem areas dorsal, lateral, or ventral to the reticular formation failed to produce significant retention deficits on either habit. Similar findings were obtained in connection with the retention of a nonvisual (kinesthetic) discrimination habit.
\end{abstract}

It is well known that the brainstem reticular formation (BSRF) is a vastly complex neural system having a superabundance of intercellular connections throughout the neuraxis (Nauta \& Kuypers, 1958; Scheibel \& Scheibel, 1958), that virtually all areas of the cerebral cortex project to this neural system (Brodal, 1958; Valverde, 1962), that stimulation. of this system exerts a powerful influence on the excitability of both higher and lower regions of the brain (Magoun, 1963), and that transection of this system at mesencephalic levels can lead to pronounced alterations in general behavioral arousal (French \& Magoun, 1952; Lindsley, Schreiner, Knowles, \& Magoun, 1950). Such a complex neural network has all the properties of an integrating or "centrencephalic" system (Fessard, 1954; Kilmer, McCulloch, \& Blum, 1968; Penfield, 1954) and could conceivably serve as the anatomical substratum for the development of the memory trace (Gastaut, 1958; John, 1967; Thompson, 1965).

It is remarkable, however, that recent lesion studies have failed to provide consistent support for the notion that the BSRF plays a fundamental role in learned behavior. For example, discrete lesions of the reticular formation (RF) at mesencephalic levels have not been found to impair learning or retention in birds (Durkovic \& Cohen, 1969), rats (Kesner, Fiedler, \& Thomas, 1967; Thompson, 1969), cats (Chow \& Randall, 1964; Myers, 1964), or in monkeys (Thompson \& Myers, 1971). In some reports using cats as Ss (Hernández-Peón \& Brust-Carmona, 1961; Myers, 1964; Sprague, Levitt, Robson, Liu, Stellar, \& Chambers, 1963), disruptive

\footnotetext{
*This study was supported in part by Grant MH 08377 from the National Institute of Mental Health. This research was done with the assistance of Samuel Craddock, Peter Spiliotos, Ted Petit, and Thomas Henderson.
}

effects on learned behavior have been observed following ventromedial mesencephalic lesions, but these effects may be more closely related to destruction of the red nucleus rather than transection of the surrounding RF (McNew, 1968; Thompson, 1969).

While these results put a strain on the hypothesis that the BSRF is intimately involved in the establishment or execution of learned responses, they are by no means conclusive. No lesion studies exist which have systematically explored the contributions of different regions of the BSRF on learning and memory. Although originally conceived as a diffuse anatomical network having common functional properties, it must now be recognized that the RF is differentially organized and may be selectively implicated in diverse physiological and behavioral functions (Bonvallet \& Newman-Taylor, 1967; Brodal, 1958; Kadjaya \& Narikashvili, 1966; Olszewski, 1954; Valverde, 1962). That this may indeed by the case with respect to the performance of learned responses in rats is suggested by the findings that lesions of the basolateral midbrain RF (nucleus cuneiformis) produce greater disturbances in retention than do lesions of the dorsolateral midbrain RF (Thompson, 1965), and that paramedial pontine RF lesions lead to greater amnestic effects than do paramedial mesencephalic RF lesions (Thompson \& Henderson, 1971).

The purpose of the present study was to examine the possibility that certain areas of the BSRF are selectively implicated in supporting learned responses in the albino rat. This was accomplished by investigating the differential effects of electrolytic lesions to various sectors of the diencephalic, mesencephalic, and pontine RF on retention of overlearned visual discrimination habits. For comparative purposes, additional groups of animals received lesions dorsal, lateral, or ventral to the BSRF. Data are also reported on the effects of RF 
lesions on retention of a nonvisual (kinesthetic) discrimination habit.

\section{METHOD}

Visual Habits

\section{Subjects}

Ninety-nine adult male albino rats of the Wistar strain served as Ss. Following training on the visual habits, 92 underwent bilateral lesions of the brainstem, while the remaining 7 constituted the normal control group. Twenty-seven operated animals died prior to the retention test, and an additional 21 were discarded from the experiment because their lesions were either minute or grossly asymmetrical.

\section{Apparatus}

A two-choice discrimination box (Thompson \& Bryant, 1955), utilizing the motive of escape (or avoidance) of footshock, was employed. Three pairs of stimulus cards were used in the apparatus. One pair (two medium gray cards) was employed in training the animals to push aside a card in order to obtain access to the goalbox. The second pair consisted of a white card and a black card (Problem WB). The third pair (Problem HV) consisted of a horizontal black and white striped card and a vertical black and white striped card, the striations being $1 / 2$ in. thick. When in use, these cards were washed several times a day and were either touched up with paint or replaced by new cards when soiled.

\section{Procedure}

Following preliminary training, all animals were first required to learn the brightness discrimination habit (Problem WB). A response to the unlocked white card (positive) admitted the animal to the goalbox, whereas a response to the locked black card (negative) was automatically punished by mild shock to the feet. Eight trials were given daily with an intertrial interval of $60 \mathrm{sec}$. The position of the positive card varied from the right to the left window in a strict double-alternation sequence. Upon reaching the criterion of learning (no more than one error in 2 consecutive days), an additional 50 overlearning trials were administered at a rate of 10 trials per day for 5 consecutive days. During overlearning, the position of the positive card followed a sequence mixed with single- and double-alternation runs.

Subsequently, the animal was trained on Problem HV: approach a horizontal black and white striped card and avoid a vertical black and white striped card. The training procedure was the same as that described for Problem WB and included 50 overlearning trials.

From 4 to $24 \mathrm{~h}$ after completing training on Problem HV, the majority of animals were subjected to brainstem lesions. Following a 2- to 3-week recovery period (or rest period in the case of the normal controls), all animals were required to relearn the two discrimination problems in the same order in which they were originally mastered. Overtraining trials (ranging from 20 to 50) were given after the criterion run was reached on Problem WB, but were not given following the attainment of the criterion run on Problem HV.

\section{Surgery}

Bilateral lesions were carried out in one stage under deep chloral hydrate anesthesia. In most instances, a constant anodal current of $1.5-2.0 \mathrm{~mA}$ for a duration of $10 \mathrm{sec}$ was passed through an implanted stainless steel electrode with $1.0 \mathrm{~mm}$ of the tip exposed. Details of the surgical procedure may be found elsewhere (Thompson, 1971).
$R F$ groups. The BSRF was arbitrarily divided into four successive frontal sectors, and different groups of rats received bilateral lesions to a given sector. Group RF-DMJ was subjected to lesions of the paramedial RF at the level of the dimesencephalic juncture (between $F 3.0 \mathrm{~mm}$ and $F 2.0 \mathrm{~mm}$ on the Massopust, 1961, atlas), Group RF-MM received lesions of the paramedial mesencephalic RF (between $F 2.0$ and $F 1.0$ ), Group RF-LM sustained lateral mesencephalic RF lesions at the same level as Group RF-MM, Group RF-MPJ suffered lesions of the paramedial $R F$ at the level of the mesencephalic-pontine juncture (between F 0.5 and F -1.0), and Group RF-P sustained lesions of the paramedial pontine RF (between $F-1.0$ and $F$ -2.0 ). The lesions were placed $1.0 \mathrm{~mm}$ lateral to the midline in all animals, except those included in Group RF-LM $(1.75 \mathrm{~mm}$ lateral). The depth of the lesions varied from 5.5 to $7.5 \mathrm{~mm}$ from the neocortical surface.

Miscellaneous groups. One group of animals (Group MISC-1) received lesions to the periaqueductal gray substance and adjacent structures (oculomotor nuclei, medial longitudinal fasciculus, and dorsal central tegmentum), while a second group (Group MISC-2) sustained lesions to brainstem areas either ventral, lateral, or dorsal to the caudal mesencephalic and/or pontine RF.

\section{Histology}

After the retention test was completed, each operated animal was anesthetized, and the brain was removed and stored in $10 \%$ Formalin for $48 \mathrm{~h}$. Each brain was then blocked, frozen, and sectioned frontally at 90 microns. Every third section through the lesion was photographed at $10-14 \mathrm{X}$ in a manner fully described elsewhere (Thompson, 1971). Description of the lesions in the text will be made in terms of the central necrotic zone (vacuolated and severely coagulated areas), which is readily delineated in.photographs of unstained sections.

\section{Measurements of Performance}

Two different error scores were calculated from the original learning and relearning records. "Criterion run" errors were defined as the number of errors committed prior to the achievement of the criterion (at least 15 correct responses within 2 successive days). "Significant run" errors were defined as the number of errors committed prior to the earliest attainment of a series of correct responses that has a probability of occurrence of less than .05 (see Thompson, 1969). From these data, a criterion run savings score and a significant run savings score were determined. Since the latter almost invariably yields a higher savings score than the former, it represents a more sensitive measure of retention. Therefore, the significant run savings score will serve as the sole index of retention, and the criterion run savings score will be reserved as an index of the animal's ability to attain a high level of response accuracy over a 2-day period.

\section{Kinesthetic Habit}

When the main experiment was completed, it was decided to determine whether those BSRF lesions which were found to impair retention of our visual discrimination habits would also impair retention of a nonvisual discrimination habit.

Fourteen adult male albino rats of the Wistar strain completed the experiment. They were initially subjected to enucleation of the eyes under deep chloral hydrate anesthesia. After a 2-day recovery period, they were trained on an incline box (kinesthetic) discrimination problem under the motive of escape from footshock to choose the arm that was inclined upward and to avoid the arm that was inclined downward. Eight trials were given daily with an intertrial interval of $60 \mathrm{sec}$. Specific details of the apparatus and training procedure may be found elsewhere 
(Thompson, Lukaszewska, Schweigerdt, \& McNew, 1967).

Following learning (at least 15 correct responses within 2 successive days), four rats were subjected to bilateral lesions of the RF at the dimesencephalic juncture (Group RF-DMJ), three sustained lesions of the mesencephalic RF located lateral to the red nucleus (Group RF-LM), three received lesions of the RF at the mes-pontine juncture (Group RF-MPJ), and the remaining four served as the normal control group. (Pontine RF lesions were not examined in the current study, but were examined in the Thompson \& Henderson, 1971, experiment.) The retention test was given 3 weeks later and consisted of relearning the kinesthetic habit.

\section{RESULTS}

\section{Visual Habits}

\section{Original Learning}

Mean criterion run and significant run errors for Problem WB were 11.9 and 10.7, while those for Problem HV were 12.5 and 10.8. With respect to criterion run and significant run days, the mean scores were 3.8 and 3.2 for Problem WB as compared to 4.4 and 3.6 for Problem HV. In terms of the Wilcoxon test for paired replicates (Siegel, 1956), none of the differences in learning scores between Problem WB and Problem HV approached statistical significance (ps>.20).

Although the two problems were learned at a comparable rate, it is important to note that the animals made significantly more overlearning errors on Problem HV (mean $=1.5)$ than on Problem WB (mean = 0.7 ), the $\mathrm{p}$ value being less than .01 .

\section{Retention and Other Behavioral Observations}

Normal control group. All seven control Ss earned perfect significant run savings scores on the two visual discrimination problems after a 2- to 3-week rest period. With respect to criterion run savings, all control animals earned perfect scores on Problem WB and only one earned an imperfect score on Problem HV (40\%).

Group RF-DMJ. Six animals received bilateral lesions

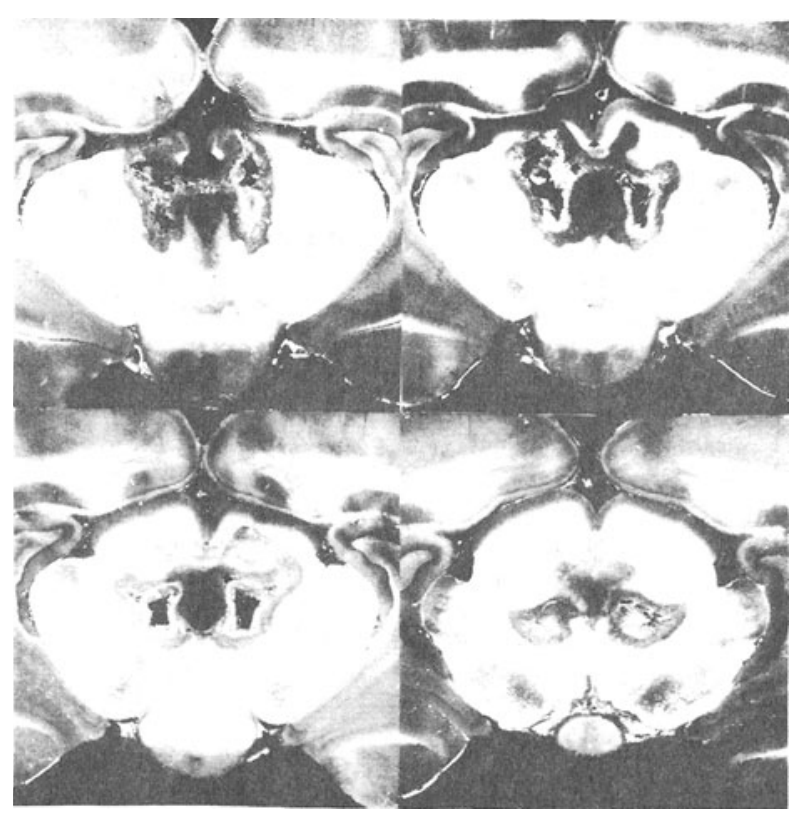

Fig. 1. Four unstained sections showing a lesion in one animal of Group RF-DMJ. (This rat earned a criterion run savings score of $-\mathbf{2 0 0 \%}$ on Problems WB and HV.)

of the medial portion of the RF at the dimesencephalic juncture. In most cases, the lesions began at the level of the anterior border of the superior colliculus and terminated at the level of the rostral pole of the red nucleus (Fig. 1). Damaged structures included the interstitial nuclei of the posterior commissure, interstitial nucleus of Cajal, parafascicular nucleus, and the lateral portion of the central gray substance. The pretectal area was slightly damaged and the habenulopeduncular tract was spared in all animals, save one.

During the first postoperative week, the majority of animals exhibited hyperextension of the neck, pupillodilation, and aphagic-adipsic difficulties. At the end of the 3-week recovery period, however, these physiological disturbances disappeared. All animals were

Table 1

Mean Retention (Percentage of Error Savings) and Overtraining Scores for All Groups

\begin{tabular}{|c|c|c|c|c|c|c|}
\hline \multirow[b]{2}{*}{ Group } & \multirow[b]{2}{*}{$\mathrm{N}$} & \multicolumn{3}{|c|}{ Problem WB } & \multicolumn{2}{|c|}{ Problem HV } \\
\hline & & SR & CR & $\begin{array}{c}\text { Overtraining } \\
\text { Percent Correct }\end{array}$ & SR & $\mathrm{CR}$ \\
\hline Control & 7 & 100 & 100 & 99.3 & 100 & 91.4 \\
\hline RF-DMJ & 6 & $66.0^{*}$ & $19.0 *$ & 97.2 & $-43.8^{*}$ & $-30.5^{*}$ \\
\hline RF-MM & 4 & 81.2 & $68.5^{*}$ & 97.5 & 97.5 & 82.5 \\
\hline RF-LM & 4 & 89.0 & $67.2 *$ & 97.0 & $10.0^{*}$ & $12.0^{*}$ \\
\hline RF-MPJ & 5 & 100 & $68.4^{*}$ & 94.8 & $54.4^{*}$ & $46.5^{*}$ \\
\hline RF-P & 4 & $62.7 *$ & $57.0^{*}$ & $90.0^{*}$ & $19.0^{*}$ & $26.0^{*}$ \\
\hline MISC-1 & 10 & 80.4 & 82.8 & 97.9 & 96.7 & 92.4 \\
\hline MISC-2 & 11 & 95.0 & 94.5 & 99.1 & 90.5 & 95.5 \\
\hline
\end{tabular}

*Differed from the controls at least at the .05 level. 


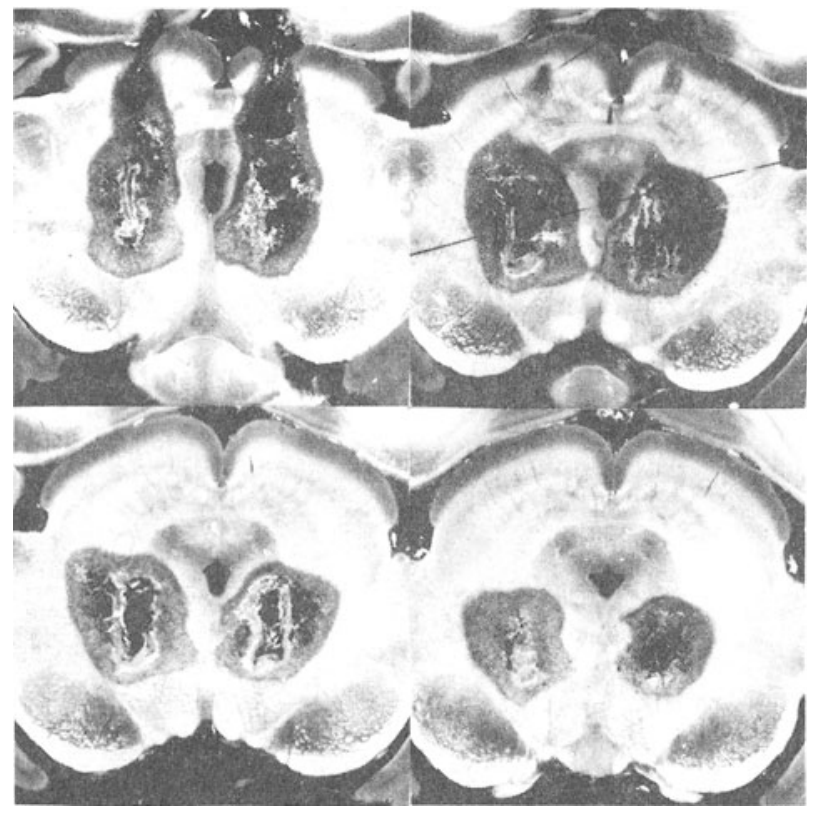

Fig. 2. Four unstained sections showing a lesion in one animal of Group RF-MM. (This rat earned criterion run savings scores of $22 \%$ and $67 \%$ on Problems WB and HV, respectively.)

found to be excellent runners in the apparatus (requiring only occasional footshocks to force choices, showing VTEs, and making adaptive correction responses following an error) during the retention test.

The Mann-Whitney test disclosed that this group was significantly inferior to the controls in retention of both Problem WB and Problem HV (see Table 1). It will also be noted that the pattern habit was impaired to a greater extent than the brightness habit: while only one animal earned a significant run savings score below 33\% on Problem WB, five earned scores below $33 \%$ on Problem HV.

Group RF-MM. The four animals of this group sustained bilateral lesions to the medial portion of the mesencephalic RF lying between the central gray substance dorsally and the prerubral and/or rubral areas ventrally (Fig. 2). In all cases, the lesions destroyed the anterodorsal half of the red nucleus bilaterally.

The only postoperative disturbances noted in these animals were a transient adipsic-aphagic disorder and a "reluctance" to use the hind legs in locomotion during the first 2-3 postsurgical days.

As noted in Table 1, this group showed a significant disturbance in reaching the criterion run on Problem WB. With respect to significant run savings scores, however, two animals showed perfect retention and the remaining two earned scores of $50 \%$ and $75 \%$. On Problem HV, three exhibited perfect retention and one earned a significant run savings score of $90 \%$.

Group RF-LM. This group of four rats sustained lesions of the mesencephalic RF lateral to the red nucleus. The lateral half of the medial lemniscus and the caudal half of the substantia nigra were damaged to varying degrees in all cases (Fig. 3).

During the first 1-2 weeks following surgery, all animals were seriously aphagic and adipsic and exhibited obstinate progression (repeatedly running off the edge of the observation table) and hyperactivity. At the time of the retention test, however, these animals appeared "normal" and were subsequently found to be excellent runners in the apparatus.

On Problem WB, these animals showed a significant disturbance in reaching the criterion run, but did not differ significantly from the controls in the attainment of the significant run. On Problem HV, all animals earned significant run and criterion run savings scores below $34 \%$.

Group RF-MPJ. Five rats received bilateral lesions of the paramedial $\mathrm{RF}$ at the mes-pontine juncture. In all cases, the brachium conjunctivum was partially interrupted (Fig. 4).

Although feeding and drinking habits were largely unimpaired, ${ }^{1}$ all animals exhibited obstinate progression, hyperactivity, hypersensitivity to footshock, and a defective labyrinthine reflex (ventroflexion of the head when the animal was suspended by its tail). Despite these disturbances, all animals were found to be excellent runners in the apparatus during the retention test.

All five animals earned perfect significant run savings scores on Problem WB. Criterion run savings scores,

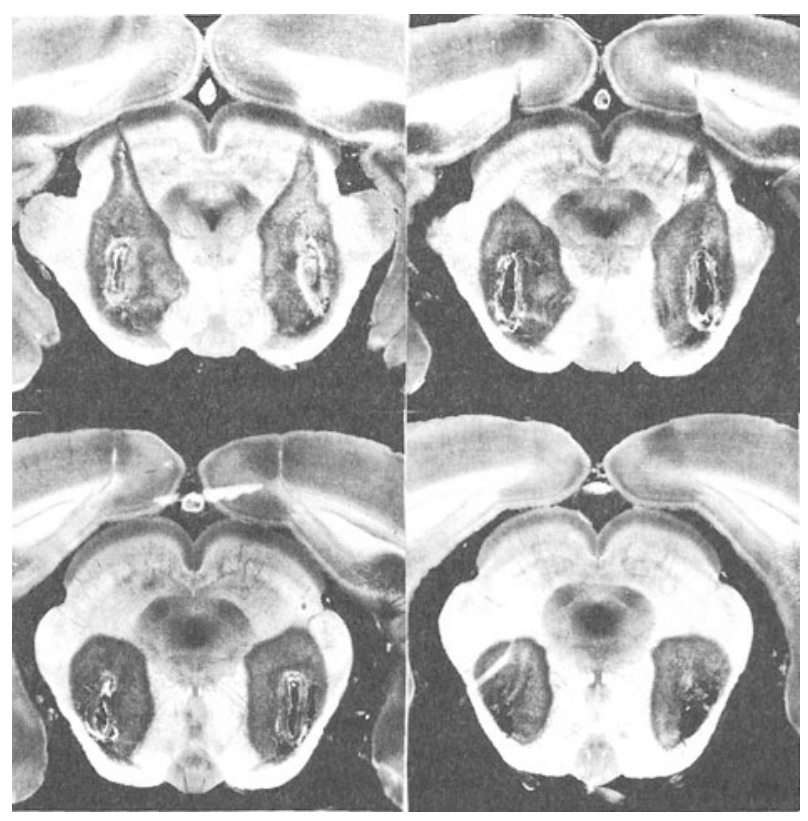

Fig. 3. Four unstained sections showing a lesion in one animal of Group RF-LM. (This rat earned criterion run savings scores of $63 \%$ and $-14 \%$ on Problems WB and HV, respectively.) 
however, were less than perfect in four animals (scores ranging from $45 \%$ to $67 \%$ ). With respect to Problem HV, one rat earned a perfect significant run savings score and the remaining four earned scores between $0 \%$ and $82 \%$.

Group RF-P. Four rats were subjected to bilateral paramedial lesions of the pontine RF between the levels of the decussating fibers of the brachium conjunctivum rostrally and the motor trigeminal nucleus caudally (Fig. 5).

During the first postoperative week, these animals were adipsic and aphagic, ataxic, hyperactive, and exhibited obstinate progression. At the time of the retention test, however, these animals appeared alert, healthy, and were subsequently found to be excellent runners in the apparatus.

As shown in Table 1, this group was significantly inferior to the controls in all measures of performance on Problems WB and HV.

Miscellaneous groups. A total of 10 animals received bilateral lesions in the region of the periaqueductal gray substance (Group MISC-1). In all cases, the lesions damaged to varying degrees the medial longitudinal fasciculus, oculomotor complex, and/or the trochlear nucleus (Fig. 6).

A variety of disturbances followed surgery, including the absence of the pupilloconstrictor reflex, defective labyrinthine reflex, obstinate progression, hyperactivity, and hypersensitivity to footshock.

As noted in Table 1, this group was not found to differ significantly from the controls in retention of

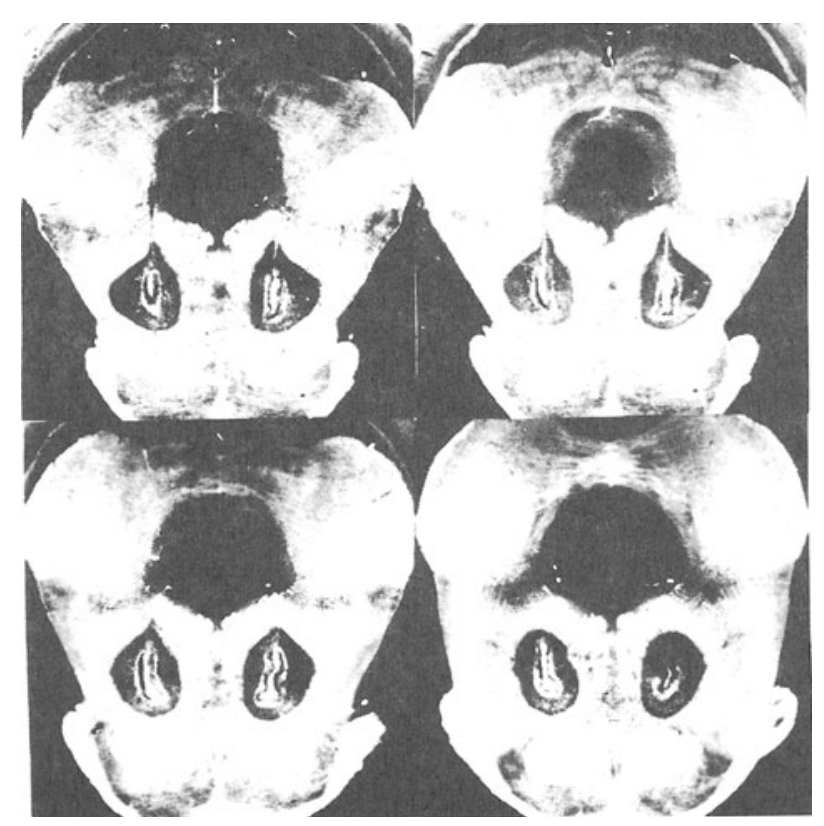

Fig. 4. Four unstained sections showing a lesion in one animal of Group RF-MPJ. (This rat earned criterion run savings scores of $45 \%$ and $70 \%$ on Problems WB and HV, respectively.)

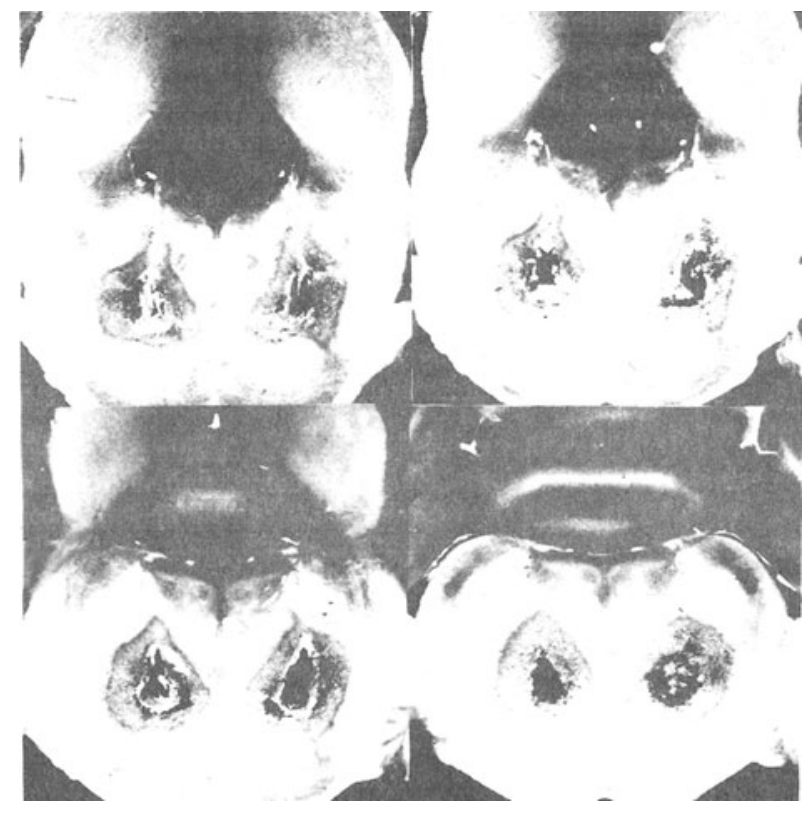

Fig. 5. Four unstained sections showing a lesion in one animal of Group RF-P. (This rat earned criterion run savings scores of $100 \%$ and $-43 \%$ on Problems WB and HV, respectively.)

either problem. Seven animals attained significant run savings scores above $90 \%$ on Problem WB and nine earned scores between $92 \%$ and $100 \%$ on Problem HV.

A second group of 11 animals (Group MISC-2) sustained bilateral lesions dorsal, lateral, or ventral to the RF at caudal mesencephalic and/or rostral pontine levels (Fig. 7). In most cases, the animals appeared "normal" by the third postsurgical day.

This group earned savings scores on both problems which were virtually indistinguishable from those of the controls. Eight showed perfect retention of Problem WB and nine showed perfect retention of Problem HV.

\section{Kinesthetic Habit}

In this experiment, the criterion run and significant run measures turned out to be very similar; therefore, only the former will be discussed.

As noted elsewhere (Thompson \& Craddock, 1972), the kinesthetic discrimination habit and the horizontal-vertical discrimination habit are comparable in difficulty. In the current study, the kinesthetic habit was learned in an average of 4.8 days and 14.6 errors.

All four control rats exhibited excellent retention of this habit, three earning perfect savings scores and one earning a score of $92 \%$. In contrast, 9 of the 10 animals with RF lesions showed poor retention of the kinesthetic habit. All four animals of Group RF-DMJ earned negative savings scores (mean savings = $-122.0 \%$ ), the three animals of Group RF-LM earned savings scores below 36\% (mean savings $=-19.3 \%$ ), and the three animals of Group RF-MP earned savings scores 


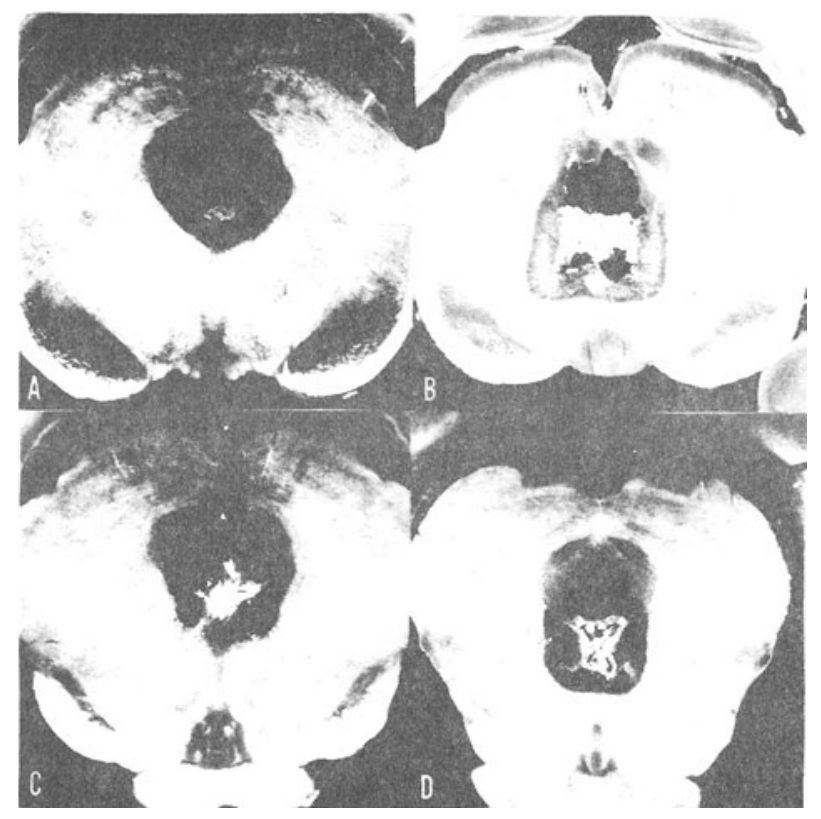

Fig. 6. Unstained sections showing lesions in four animals composing Group MISC-1. (All four rats earned $100 \%$ criterion run savings scores on Problems WB and HV.)

of $-200 \%, 10 \%$, and $100 \%$. The Mann-Whitney test disclosed that the former two operated groups were significantly inferior to the controls in retention of the kinesthetic habit.

\section{Summary}

As noted in Table 1, Problem HV was more vulnerable than Problem WB to the deleterious effects of BSRF lesions. Of the 23 animals sustaining lesions to the RF, 17 earned poorer significant run savings scores on Problem HV than on Problem WB, 2 showed perfect retention of both habits, and 4 earned poorer scores on Problem WB than on Problem HV. This is to be contrasted with the performance of the control group (all 7 rats showed perfect retention of both habits) and with the performance of those Ss (Groups MISC-1 and MISC-2) receiving lesions peripheral to the BSRF-of the 21 rats involved, 4 earned poorer significant run savings scores on Problem HV than on Problem WB, 11 showed perfect retention of both habits, and 6 earned poorer scores on Problem WB than on Problem HV. These differences, according to the chi-square test, are significant beyond the .01 level.

Figure 8 presents a summary of some of the major findings of this experiment. The left side of each section shows the distribution of lesions in those rats exhibiting a conspicuous retention loss on Problem HV, those Ss earning significant run savings scores below 33\%. The right side of each section shows the distribution of lesions in those rats exhibiting a conspicuous retention loss on the kinesthetic habit (significant run savings

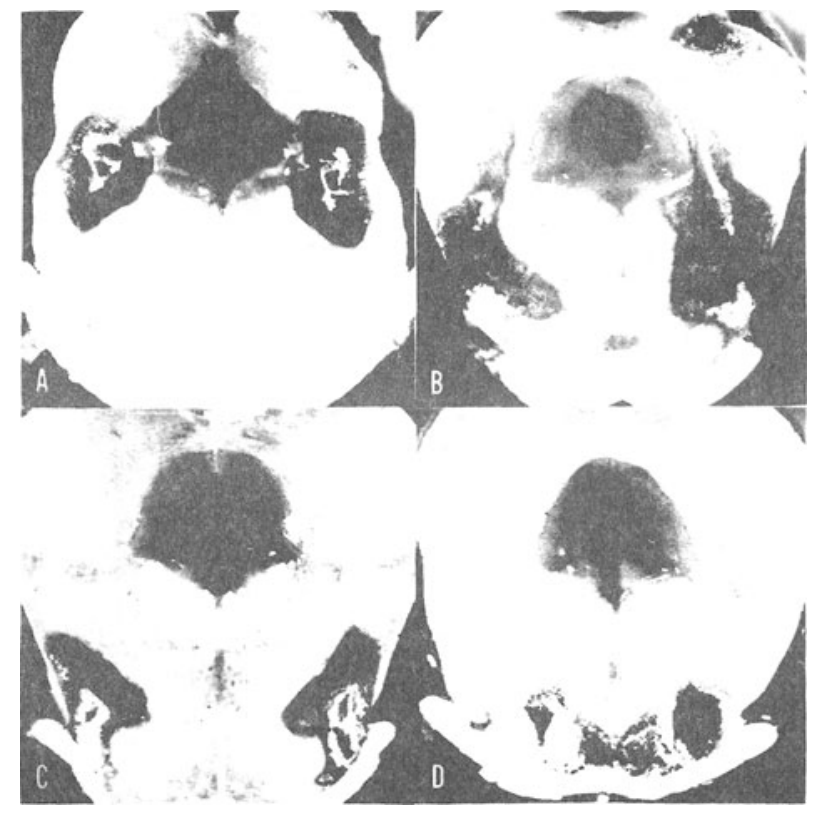

Fig. 7. Unstained sections showing lesions in four animals composing Group MISC-2. (All four rats earned 100\% criterion run savings scores on Problems WB and HV.)

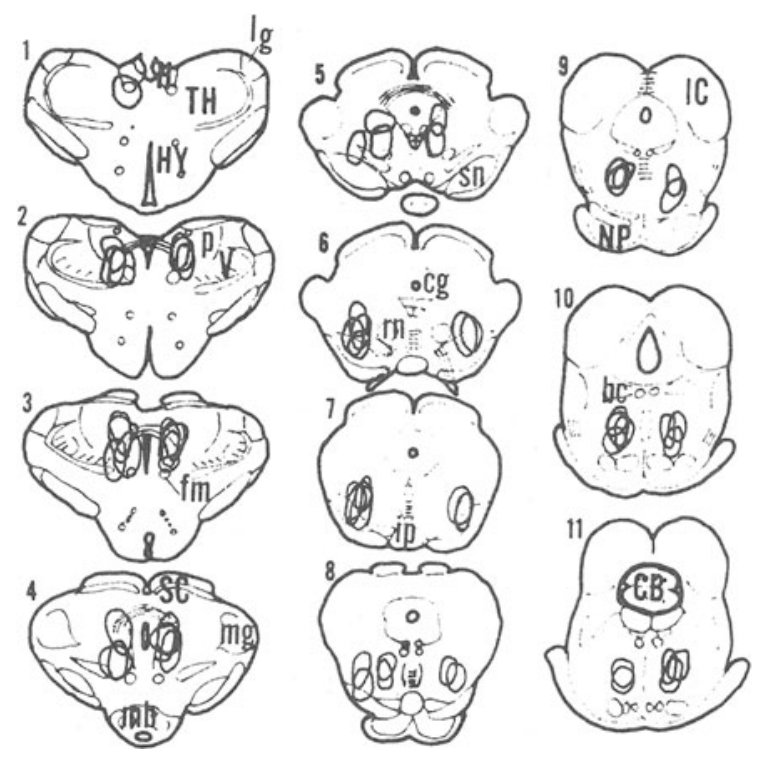

Fig. 8. Semidiagrammatic composites of lesions at 11 levels of the brainstem. (Enclosed areas on the left side of each section indicate placement of lesions yielding a loss in retention of Problem HV, while those on the right side of each section indicate placement of lesions yielding a loss in retention of the kinesthetic problem. Abbreviations: bc $=$ brachium conjunctivum; $\mathrm{C}=$ cerebellum; $\mathrm{cg}=$ central gray; $\mathrm{fm}=$ habenulopeduncular tract; $\mathbf{H}=$ habenula; $H Y=$ hypothalamus; IC = inferior colliculus; ip = interpeduncular nucleus; $l g$ = lateral geniculate nucleus; $\mathrm{mg}=$ medial geniculate nucleus; $\mathrm{mm}=$ mamillary bodies; $p=$ pretectal area; $P=$ pons; $m=$ red nucleus; $\mathrm{SC}=$ superior colliculus $; \mathrm{sn}=$ substantia nigra $; \mathrm{TH}=$ thalamus; $\mathrm{V}$ $=$ ventral thalamic nucleus.) 
scores $<33 \%$ ). (The pontine RF lesions shown on the right side were taken from the Thompson and Henderson, 1971, study.) Inspection of this figure reveals that those areas of the BSRF that are critical for retention of a visual discrimination habit are likewise critical for retention of a nonvisual kinesthetic discrimination habit.

\section{DISCUSSION}

\section{General Findings}

The overall results of this study clearly show that the integrity of certain portions of the BSRF is necessary for the maintenance of visual as well as nonvisual discrimination habits in the albino rat. Critical regions were found at all levels of the neuraxis studied, including the dimesencephalic juncture, the mesencephalon, and the rostral metencephalon (see Fig. 8). According to the nomenclature used by Skinner (1971), the critical regions lie within the nucleus cuneiformis (particularly the rostral extension of this nucleus at the dimesencephalic juncture, the basolateral portion of this nucleus at mesencephalic levels, and the caudal extension of this nucleus at the mes-metencephalic juncture) and the nucleus reticularis pontis oralis. The only region of the RF that was not found to be essential for normal retention of brightness or pattern discrimination habits was the area dorsal to the red nucleus (the dorsal portion of the nucleus cuneiformis at mesencephalic levels).

These results confirm certain earlier reports dealing with visual discrimination performance in the brainstem-damaged rat. Thus, the finding that lesions of the rostral extension of the nucleus cuneiformis impair retention of visual habits is consistent with the results of Thompson (1969), Thompson, Truax, and Thorne (1970), and Thorne (1970). Similarly, the disruptive effects on visual habits following lesions of either the basolateral portion of the nucleus cuneiformis or the nucleus reticularis pontis oralis have previously been observed by Thompson (1965) and Thompson and Henderson (1971). Finally, the absence of deleterious effects on simple brightness or pattern discrimination habits following lesions of the dorsal mesencephalic RF is in accord with the results of McNew (1968), Thompson (1963, 1969), Thompson and Massopust (1960), and Thompson, Rich, and Langer (1964).

\section{Conflicting Reports}

Numerous lesion studies have been reported which, at first glance, strongly militate against the notion that the BSRF plays an important role in learning and memory. From our own laboratory, for example, data have been reported which question the significance of the basolateral (Thompson, 1963, 1969; Thompson \& Massopust, 1960) and caudal (Thompson \& Massopust,
1960; Thompson et al, 1964) portions of the nucleus cuneiformis in the execution of discriminative responses to visual cues. Other lesion studies discrediting the functional significance of the BSRF in learning and memory include Durkovic and Cohen (1969) using birds, Kesner et al (1967) using rats, Chow and Randall (1964) using cats, and Thompson and Myers (1971) using monkeys.

The existence of critical foci within the BSRF for visual (and kinesthetic) discrimination deficits would appear to provide a satisfactory basis for explaining, at least in part, these discrepant results. This explanation is by no means farfetched when it is considered that critical foci for visual discrimination deficits have already been reported for the red nucleus area (McNew, 1968; Thompson et al, 1964), the posterior thalamus (Breen, 1965; McNew \& Thompson, 1966; Thompson \& Rich, 1961), and the interpedunculo-central tegmental area (Craddock \& Thompson, 1971). Another variable which may be partly responsible for the contradictory reports is the difficulty of the habit learned (or relearned) by the brainstem-damaged animals. According to the analysis made by McNew (1968), lesions of the dorsal mesencephalic RF impair performance of difficult visual discrimination habits, while leaving simple visual discrimination habits intact. This observation, made by McNew, has since been confirmed (see the performance of the subcollicular group in Thompson, 1969), and may well apply to lesions in other parts of the RF. In the current study, for example, Problem HV was found to be considerably more susceptible to interference by BSRF lesions than Problem WB. Still another possible variable contributing to discrepancies in the literature over the functional significance of the BSRF in learning and memory lies in the manner in which the lesions are produced. In the present study, all Ss sustained one-stage rather than multiple-stage lesions of the RF. This difference in surgical procedure has been considered by Chow and Randall (1964) and Kesner et al (1967) to be a prime factor in influencing the extent to which behavioral deficits follow RF lesions. ${ }^{2}$ Finally, the size of the lesions investigated cannot be ignored as a possible contributory factor to the conflicting reports. According to the work of Majkowski, Sobieszek, Kowalski, and Szwed (1970), extensive damage to the mesencephalic RF leads to dramatic losses in acquisition of conditioned responses in cats despite the fact that the surgery was performed in two stages.

\section{A General Interpretation}

Before discussing the significance of these findings, it is important to provide a conceptual framework within which the results of a long series of lesion experiments on retention of learned responses can be interpreted. Despite its limitations and pitfalls, the lesion method still constitutes one of the most straightforward and reliable ways to identify those parts of the brain that are 
essential for the expression (memory) of previously learned responses. Granting this fact, it is possible to map the "memory system" of the brain for any given learned response simply by canvassing many different cortical and subcortical areas with small lesions (see Thompson, 1969). Furthermore, by mapping specific memory systems for a variety of learned responses, the "general memory system" of the brain can be identified-those anatomical areas in which the specific memory systems overlap.

It is becoming increasingly clear, at least with respect to the rat, that the nucleus posterior thalami, posterolateral hypothalamus, red nucleus, substantia nigra, interpedunculocentral tegmental area, and the critical reticular regions depicted in Fig. 8 compose the general memory system of the brain. This is suggested by the findings that the only lesions which impair retention of visual discrimination habits, a kinesthetic discrimination habit, and an avoidance conditioned response to either auditory or visual signals distribute themselves within the foregoing brainstem areas (Thompson, Duke, Malin, \& Hawkins, 1961; Thompson \& Hawkins, 1961; Thompson, Lukaszewska, Schweigerdt, \& McNew, 1967; Thompson et al, 1964). Additional support for this notion comes from two studies recently completed at the Louisiana State University laboratory. Specifically, it was found that retention of an assortment of latch box problems (using the thirst motive) as well as a simple maze habit (using the pain-avoidance motive) is totally abolished following damage to either the posterior diencephalon, ventral mesencephalon, or pontine RF.

On the strength of these data, we are led to speculate that the above-mentioned brainstem structures make up the major part of the general memory system in the rat. Furthermore, since these brainstem structures occupy regions which roughly parallel the trajectory of the ascending reticular activating system (Moruzzi \& Magoun, 1949) and conspicuously overlap the brainstem reticular core (Scheibel \& Scheibel, 1958) as well as the cholinergic reticular system (Shute \& Lewis, 1967), the possibility must be considered that the general memory system is coextensive with the BSRF.

These findings on the possible identification of the general memory system (GMS) are of interest for a number of reasons. First, this system shows a remarkably high degree of localization, occupying no more than $1 / 10$ of the total volume of the brain. It should be obvious that these findings are incompatible with the view that the capacity to perform any given learned response is dependent upon the amount of cortical (and/or subcortical) tissue and not upon its anatomical localization (John, 1967; Lashley, 1950). Second, with the possible exception of the interpeduncular nucleus and the posterolateral hypothalamus, no major limbic structure comprises the GMS. This is a conspicuous omission in view of the vast amount of research implicating the limbic system in learning (recent memory), emotions, motivation, and other biologically important activities (see MacLean, 1970). Nevertheless, there is strong evidence showing that extensive damage to either the cingulate cortex, hippocampus, septal area, amygdala, or mamillary bodies fails to interfere with the execution of previously learned visual discrimination habits (Thompson, 1969). Third, no neocortical area is included within the GMS. This, of course, does not imply that various divisions of the neocortex are totally deficient of functions which support previously learned habits. The occipital cortex is clearly part of the "visual" memory system (Lashley, 1950; Thompson, 1969), and the sensorimotor cortex has been reasonably well established to be part of the "kinesthetic" memory system (Lashley, 1929; Thompson, Malin, \& Hawkins, 1961). The important point to be made, however, is that no specific cortical area seems to be essential for the expression of both visual and kinesthetic habits. Fourth, those areas of the brain traditionally regarded as having significant sensorimotor functions, such as the motor cortex, corpus striatum, and cerebellum, have not been demonstrated to be components of the GMS (Thompson, 1969). Such a finding suggests that the BSRF may provide descending pathways which are prerequisite for the initiation and control of learned responses.

The proposed participation of the BSRF in supporting the execution of all learned responses raises the question concerning the functional pathways interconnecting the brainstem reticular core with the neocortex. Although crucial evidence on this matter is lacking, the results of certain lesion studies (Howze \& Thompson, 1972; Thompson, 1969; Thompson \& Craddock, 1972) provide the basis for constructing the hypothesis that the cephalic extension of the BSRF through the lateral hypothalamic-subthalamic-internal capsule route (Scheibel \& Scheibel, 1958) and the corticoreticular systems descending through the internal capsule-cerebral peduncle-substantia nigra route (Knook, 1966; Valverde, 1962) constitute the important reciprocal links between the BSRF and the neocortex. This notion, that the expression of any learned response is dependent upon the activity of a cortico-reticulo-cortical circuit, is currently under investigation.

With regard to the functional significance of the BSRF in the performance of learned responses, it must be emphasized at the outset that the amnestic effects induced by BSRF lesions are not entirely due to a reduction in "tonic" (Lashley, 1954) or "energizing" (Luria, 1970) influences exerted upon the neocortex by the brainstem. The lesions investigated in the current study were small and failed to produce somnolence in any of our Ss. According to the work of Doty, Beck, and Kooi (1959), extensive lesions of the mesencephalic RF are required in order to produce persistent high voltage, slow wave patterns on the EEG, and even then the effect reverses in time. Moreover, lesions damaging that 
mesencephalic site (suprarubral area), which has been reported to be a focal point of the ascending reticular activating system (Moruzzi \& Magoun, 1949), do not produce retention losses on simple visual and nonvisual discrimination tasks (McNew, 1968; Thompson, 1969; Thompson et al, 1964; current study).

Perhaps the most persuasive evidence favoring the notion that the BSRF plays a central role in learning and memory comes from the recent neurophysiological studies by Olds and his associates (Disterhoft \& Olds, 1972; Olds, Disterhoft, Segal, Kornblith, \& Hirsh, 1972) on the identification of those units of the brain that exhibit "conditioned responses" with the shortest latencies. Although these units were identified at various levels of the neuroaxis, their distribution within the brainstem remarkably coincides with the critical reticular areas shown in Fig. 8. Specifically, these "learning units" were found within the pontine RF, the caudal, basolateral, and rostral extensions of the nucleus cuneiformis, and the nucleus posterior thalami. No such units were found in the superior colliculus or subcollicular tegmentum. These results parallel our findings to such an extent that the possibility of engrammic formation within the BSRF must not be overlooked.

If subsequent studies confirm the central role of the BSRF in learned behavior, then the question arises concerning the degree to which each part of the BSRF has a specialized function. Conceivably, one component may play a role in the motivational process, a second in the attentional process, a third in the retrieval process, a fourth in motor control, etc. Alternatively, all components of the BSRF may contribute to the establishment and execution of learned responses in a manner which can only be expressed in terms of dynamic interactions between the various parts. Examples of the latter would include "centrencephalic" (Penfield, 1954) or "command" (Kilmer et al, 1968) functions, "central facilitory set" (Sperry, 1955), "suppression of error-producing tendencies" (Harlow, 1959), and the like. A solution to this problem may come from a comparative analysis of either the symptomotology arising from lesions to different parts of the BSRF or the sequential order of events occurring in "learning units" localized at different sites within the BSRF.

\section{REFERENCES}

Bonvallet, M., \& Newman-Taylor, A. Neurophysiological evidence for a differential organization of the mesencephalic reticular formation. Electroencephalography \& Clinical Neurophysiology, 1967, 22, 54-73.

Breen, T. E. The effect of occipital and pretectal lesions on retention of easy and difficult brightness discriminations in the rat. Unpublished doctoral dissertation, Louisiana State University, 1965.

Brodal, A. The reticular formation of the brain stem. Springfield, Ill: Thomas, 1958.

Chow, K. L., \& Randall, W. Learning and retention in cats with lesions in reticular formation. Psychonomic Science, 1964, 1, 259-260.

Craddock, S. N., \& Thompson, R. A discrete interpedunculo-central tegmental region critical for retention of visual discrimination habits in the white rat. Journal of Comparative \& Physiological Psychology, 1971, 76, 39-50.

Disterhoft, J. F., \& Olds, J. Differential development of conditioned unit changes in thalamus and cortex of rat. Journal of Neurophysiology, 1972, 35, 665-679.

Doty, R. W., Beck, E. C., \& Kooi, K. A. Effect of brainstem lesions on conditioned responses of cats. Experimental Neurology, 1959, 1, 360-385.

Durkovic, R. G., \& Cohen, D. H. Effect of rostral midbrain lesions on conditioning of heart- and respiratory-rate responses in pigeons. Journal of Comparative \& Physiological Psychology, 1969, 68, 184-192.

Fessard, A. E. Mechanisms of nervous integration and conscious experience. In J. F. Delafresnaye (Ed.), Brain mechanisms and consciousness. Springfield, Ill: Thomas, 1954.

French, J. D., \& Magoun, H. W. Effects of chronic lesions in central cephalic brain stem of monkeys. Archives of Neurology \& Psychiatry, 1952, 68, 591-604.

Gastaut, H. The role of the reticular formation in establishing conditioned responses. In H. H. Jasper (Ed.), Reticular formation of the brain. Boston: Little, Brown, 1958.

Harlow, H. F. Learning set and error factor theory. In S. Koch (Ed.), Psychology: A study of a science. New York: McGraw-Hill, 1959.

Hernández-Peón, R., \& Brust-Carmona, H. Functional role of subcortical structures in habituation and conditioning. In J. F. Delafresnaye (Ed.), Brain mechanisms and learning. Oxford: Blackwell, 1961.

Howze, M. A., \& Thompson, R. Disconnecting occipitoreticular projections: Amnestic effects on a visual habit in the rat. Psychonomic Science, 1972, 27, 143-145.

John, E. R. Mechanisms of memory. New York: Academic Press, 1967.

Kadjaya, D. V., \& Narikashvili, S. P. Topography of electrical responses to different peripheral stimuli in the mesencephalic reticular formation of the cat. Physiology \& Behavior, 1966, 1, 209-213.

Kesner, R. P., Fiedler, P., \& Thomas, G. J. Function of the midbrain reticular formation in regulating level of activity and learning in rats. Journal of Comparative \& Physiological Psychology, 1967, 63, 452-457.

Kilmer, W. L., McCulloch, W. S., \& Blum, J. An embodiment of some vertebrate command and control principles. Currents in Modern Biology, 1968, 2, 81-97.

Knook, H. L. The fiber-connections of the forebrain. Philadelphia: Davis, 1966.

Lashley, K. S. Brain mechanisms and intelligence. Chicago: University of Chicago Press, 1929.

Lashley, K. S. In search of the engram. Symposia of the Society for Experimental Biology, 1950, No. IV, 454-482.

Lashley, K. S. Dynamic processes in perception. In J. F. Delafresnaye (Ed.), Brain mechanisms and consciousness. Springfield, Ill: Thomas, 1954.

Lindsley, D. B., Schreiner, L. H., Knowles, W. B., \& Magoun, H. W. Behavioral and EEG changes following chronic brain stem lesions in the cat. Electroencephalography \& Clinical Neurophysiology, 1950, 2, 483-498.

Luria, A. R. The functional organization of the brain. Scientific American, 1970, 222, 66-78.

Magoun, H. W. The waking brain. Springfield, Ill: Thomas, 1963.

Majkowski, J., Sobieszek, A., Kowalski, J., \& Szwed, M. EEG and behavioral correlates of learning in cats with lesions in the mesencephalic reticular formation. Brain Research, 1970, 21, 301-304.

MacLean, P. The triune brain, emotion, and scientific bias. In F. O. Schmitt (Ed.), The neurosciences. New York: Rockefeller University Press, 1970. 
McNew, B. R., \& Thompson, R. Effect of posterior thalamic lesions on retention of a brightness discrimination motivated by thirst. Journal of Comparative \& Physiological Psychology, 1966, 62, 125-128.

McNew, J. J. Role of the red nucleus in visually guided behavior in the rat. Journal of Comparative \& Physiological Psychology, 1968, 65, 282-289.

Moruzzi, G., \& Magoun, H. W. Brain stem reticular formation and activation of the EEG. Electroencephalography \& Clinical Neurophysiology, 1949, 1, 455-473.

Myers, R. E. Visual deficits after lesions of brain stem tegmentum in cats. Archives of Neurology, 1964, 11, 73-90.

Nauta, W. J. H., \& Kuypers, H. G. J. M. Some ascending pathways in the brain stem reticular formation. In H. H. Jasper (Ed.), Reticular formation of the brain. Boston: Little, Brown, 1958.

Olds, J., Disterhoft, J. F., Segal, M., Kornblith, C. L., \& Hirsh, R. Learning centers of rat brain mapped by measuring latencies of conditioned unit responses. Journal of Neurophysiology, 1972, 35, 202-219.

Olszewski, J. The cytoarchitecture of the human reticular formation. In J. F. Delafresnaye (Ed.), Brain mechanisms and consciousness. Springfield, Ill: Thomas, 1954.

Penfield, W. Studies of the cerebral cortex of man: A review and an interpretation. In J. F. Delafresnaye (Ed.), Brain mechanisms and consciousness. Springfield, I1l: Thomas, 1954.

Scheibel, M. E., \& Scheibel, A. B. Structural substrates for integrative patterns in the brain stem reticular core. In $\mathrm{H}$. H. Jasper (Ed.), Reticular formation of the brain. Boston: Little, Brown, 1958.

Shute, C. C. D., \& Lewis, P. R. The ascending cholinergic reticular system: Neocortical, olfactory and subcortical projections. Brain, 1967, 90, 497-520.

Siegel, S. Nonparametric statistics for the behavioral sciences. New York: McGraw-Hill, 1956.

Skinner, J. E. Neuroscience: A laboratory manual. Philadelphia: Saunders, 1971.

Sperry, R. W. On the neural basis of the conditioned response. British Journal of Animal Behaviour, 1955, 3, 41-44.

Sprague, J. M., Levitt, M., Robson, K., Liu, C. N., Stellar, E., \& Chambers, W. W. A neuroanatomical and behavioral analysis of the syndromes resulting from midbrain lemniscal and reticular lesions in the cat. Archives Italiennes de Biologie, 1963, 101, 225-295.

Thompson, R. Cortical and subcortical structures mediating visual discrimination habits in the rat. Boletin del Instituto de estudios Medicos y Biologicos, Universidad nacional de Mexico, 1963, 21, 451-466.

Thompson, R. Centrencephalic theory and interhemispheric transfer of visual habits. Psychological Review, 1965, 72, 385-398.

Thompson, R. Localization of the "visual memory system" in the white rat. Journal of Comparative \& Physiological Psychology Monograph, 1969, 69, 1-29.

Thompson, R. Introducing subcortical lesions by electrolytic methods. In R. D. Myers (Ed.), Methods in psychobiology. New York: Academic Press, 1971.

Thompson, R., \& Bryant, J. H. Memory as affected by activity of the relevant receptor. Psychological Reports, 1955, 1, $393-400$.

Thompson, R., \& Craddock, S. N. Lateral cerebral peduncle lesions: Amnestic effects on learned habits in the rat.
Psychonomic Science, 1972, 27, 140-142.

Thompson, R., Duke, R. B., Malin, C. F., \& Hawkins, W. F. The interpeduncular nucleus and retention in albino rats. Journal of Comparative \& Physiological Psychology, 1961, 54, 329-333.

Thompson, R., \& Hawkins, W. F. Memory unaffected by mamillary body lesions in the rat. Experimental Neurology, 1961, 3, 189-196.

Thompson, R., \& Henderson, T. C. Pontine reticular formation lesions: Amnestic effects on learned habits in the rat. Psychonomic Science, 1971, 25, 169-170.

Thompson, R., Lukaszewska, I., Schweigerdt, A., \& McNew, J. J. Retention of visual and kinesthetic discriminations in rats following pretecto-diencephalic and ventral mesencephalic damage. Journal of Comparative \& Physiological Psychology, $1967,63,458-468$.

Thompson, R., Malin, C. F., \& Hawkins, W. F. Effect of subcortical lesions on retention of a kinesthetic discrimination habit. Experimental Neurology, 1961, 3, 367-374.

Thompson, R., \& Massopust, L. C. The effect of subcortical lesions on retention of a brightness discrimination in rats. Journal of Comparative \& Physiological Psychology, 1960, 53, 488-496.

Thompson, R., \& Myers, R. E. Brainstem mechanisms underlying visually guided responses in the rhesus monkey. Journal of Comparative \& Physiological Psychology, 1971, 74, 479-512.

Thompson, R., \& Rich, I. A discrete diencephalic pretectal area critical for the retention of visual habits in the rat. Experimental Neurology, 1961, 4, 436-443.

Thompson, R., Rich, I., \& Langer, S. K. Lesion studies on the functional significance of the posterior thalamo-mesencephalic tract. Journal of Comparative Neurology, 1964, 123, 29-44.

Thompson, R., Truax, T., \& Thorne, M. Retention of visual learning in the white rat following knife-cuts through the posterior thalamus and ventromedial midbrain. Brain, Behavior \& Evolution, 1970, 3, 261-284.

Thorne, M. Visual discrimination performance in rats following nucleus posterior thalami and di-mesencephalic juncture damage. Journal of Comparative \& Physiological Psychology, $1970,71,136-146$.

Valverde, F. Reticular formation of the albino rat's brain stem cytoarchitecture and corticofugal connections. Journal of Comparative Neurology, 1962, 119, 25-53.

\section{NOTES}

1. The animals of this group intentionally received smaller lesions than those composing the other RF groups due to a high mortality rate. The absence of aphagic-adipsic disturbances (and smaller retention deficits) probably reflect a difference in lesion size rather than lesion locus.

2. No systematic study has been published which has demonstrated that one-stage lesions of the RF produce significantly greater deficits in learning or memory than two-stage RF lesions. Investigations of other brainstem areas, such as the nucleus posterior thalami (Thorne, 1970) and the red nucleus-substantia nigra area (Thompson, 1969) have consistently failed to show that this difference in surgical procedure is a significant variable.

(Received for publication October 25, 1972; revision received December 7,1972 .) 